\title{
PARACOMPACTNESS WITH RESPECT TO AN IDEAL
}

\section{T. R. HAMLETT}

Department of Mathematics East Central University Ada, Oklahoma USA 74820

\section{DAVID ROSE}

Southeastern College of the Assemblies of God 1000 Longfellow Blvd. Lakeland, Florida USA 35801

\section{DRAGAN JANKOVIĆ}

Dept. of Mathematical Sciences Cameron University Lawton, Oklahoma USA 73505

(Received May 26, 1995 and in revised form October 26,1995)

\begin{abstract}
An ideal on a set $\mathrm{X}$ is a nonempty collection of subsets of $\mathrm{X}$ closed under the operations of subset and finite union. Given a topological space $\mathrm{X}$ and an ideal $\mathcal{I}$ of subsets of $\mathrm{X}, \mathrm{X}$ is defined to be I-paracompact if every open cover of the space admits a locally finite open refinement which is a cover for all of $\mathrm{X}$ except for a set in $\mathcal{I}$. Basic results are investigated, particularly with regard to the $\mathcal{I}$ paracompactness of two associated topologies generated by sets of the form $U-I$ where $U$ is open and $I \in \mathcal{I}$ and $U\{U \mid U$ is open and $U-A \in I$, for some open set $A\}$. Preservation of $\mathcal{I}$-paracompactness by functions, subsets, and products is investigated. Important special cases of $\mathcal{I}$-paracompact spaces are the usual paracompact spaces and the almost paracompact spaces of Singal and Arya ["On mparacompact spaces", Math. Ann., 181 (1969), 119-133]
\end{abstract}

KEY WORDS AND PHRASES: ideal, compact, paracompact, H-closed, quasi-H-closed, nowhere dense, meager, (continuous, almost continuous, open, closed, perfect) functions, regular closed, open cover, refinement, locally finite family, $\tau$-boundary ( $\tau$-codense) ideal, compatible ( $\tau$-local) ideal.

AMS SUBJECT CLASSIFICATION: 54D18, 54D30

\section{INTRODUCTION}

The concept of paracompactness with respect to an ideal was introduced by Zahid in [1] The concepts of almost paracompactness [2] of Singal and Arya and para-H-closedness of Zahid [1] are special cases

An ideal on a set $\mathrm{X}$ is a nonempty collection of subsets of $\mathrm{X}$ closed under the operations of subset ("heredity") and finite union ("finite additivity"). An ideal closed under countable unions ("countable

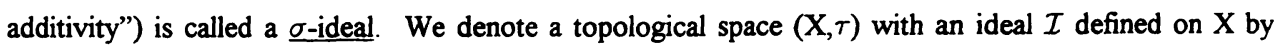
$(\mathrm{X}, \tau, \mathcal{I})$. Given a space $(\mathrm{X}, \tau)$ and $\mathrm{A} \subseteq \mathrm{X}$, we denote by $\operatorname{Int}_{\tau}(\mathrm{A})$ and $\mathrm{Cl}_{\tau}(\mathrm{A})$ the interior and closure of $\mathrm{A}$, respectively, with respect to $\tau$. When no ambiguity is present we write simply $\operatorname{Int}(\mathrm{A})$ and $\mathrm{Cl}(\mathrm{A})$. If $x \in X$, we denote the open neighborhood system at $x$ by $\tau(x)$; i e., $\tau(x)=\{U \in \tau \mid x \in U\}$. We abbreviate "if and only if" with "iff" The conclusion or omission of a proof is designated by the symbol " $\square$ "

\section{BASIC RESULTS}

Let us begin with the following definition.

DEFINITION [1] A space $(\mathrm{X}, \tau, \mathcal{I})$ is said to be $\mathcal{I}$-paracompact, or paracompact with respect to $\mathcal{I}$, iff every open cover $\Gamma$ of $X$ has a locally finite open refinement $\gamma$ (not necessarily a cover) such that $X$ -

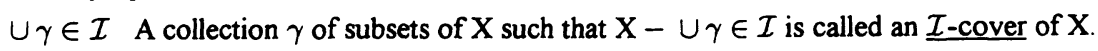

Singal and Arya [2] define a space $(X, \tau)$ to be almost paracompact if every open cover $\Gamma$ of $X$ has a locally finite refinement $\gamma$ such that $\mathrm{X}=\mathrm{Cl}(\cup \gamma)$. Zahid [1] defines a space to be para-H-closed if it is almost paracompact and $\mathrm{T}_{2}$. 
Given a space $(\mathrm{X}, \tau)$, we denote by $\mathcal{N}(\tau)$ the ideal of nowhere dense subsets of $(\mathrm{X}, \tau)$ The following theorem establishes that almost paracompactness and para-H-closedness are special cases of $\mathcal{I}$ paracompactness.

THEOREM II.1. (1) A space $(\mathrm{X}, \tau)$ is almost paracompact iff $(\mathrm{X}, \tau)$ is $\mathcal{N}(\tau)$-paracompact

(2) [1] A $\mathrm{T}_{2}$ space $(\mathrm{X}, \tau)$ is para-H-closed iff $(\mathrm{X}, \tau)$ is $\mathcal{N}(\tau)$-paracompact.

The following obvious result is stated for the sake of completeness

THEOREM II.2. If $(\mathrm{X}, \tau, \mathcal{I})$ is $\mathcal{I}$-paracompact and $\mathcal{J}$ is an ideal on $\mathrm{X}$ such that $\mathcal{I} \subseteq \mathcal{J}$, then $(\mathrm{X}, \tau, \mathcal{J})$ is $\mathcal{J}$-paracompact.

Given a space $(\mathrm{X}, \tau, \mathcal{J})$, the collection $\beta(\mathcal{I}, \tau)=\{\mathrm{U}-\mathrm{I}: \mathrm{U} \in \tau, \mathrm{I} \in \mathcal{I}\}$ is a basis for a topology $\tau^{*}(\mathcal{I})$ finer than $\tau$ [3]. When no ambiguity is present we denote $\beta(\mathcal{I}, \tau)$ by $\beta$ and $\tau^{*}(\mathcal{I})$ by $\tau^{*}$. If $\beta=\tau^{*}$, then we say $\mathcal{I}$ is $\tau$-simple. A sufficient condition for $\mathcal{I}$ to be simple is the following: for $\mathrm{A} \subseteq \mathrm{X}$; if for every $\mathrm{a} \in \mathrm{A}$ there exists $\mathrm{U} \in \tau(\mathrm{a})$ such that $\mathrm{U} \cap \mathrm{A} \in \mathcal{I}$, then $\mathrm{A} \in \mathcal{I}$. If $(\mathrm{X}, \tau, \mathcal{I})$ satisfies this condition, then $\tau$ is said to be compatible with respect to $\mathcal{I}$ [4] or $\mathcal{I}$ is said to be $\tau$-local, denoted $\mathcal{I} \sim \tau$. If $(\mathrm{X}, \tau)$ is an infinite discrete space, then the ideal of finite sets is $\tau$-simple but not $\tau$-local. It is known that $\mathcal{N}(\tau) \sim \tau$ in any space [5]. It is also known [Banach Category Theorem, 6] that $\mathcal{M}(\tau) \sim \tau$ in any space where $\mathcal{M}(\tau)$ denotes the $\sigma$-ideal of meager (or first category) subsets.

Given a space $(\mathrm{X}, \tau, \mathcal{I})$ and $\mathrm{A} \subseteq \mathrm{X}$, we denote by $\mathrm{A}^{*}(\mathcal{I}, \tau)$, or simply $\mathrm{A}^{*}$ when no ambiguity is present, the following: $\mathrm{A}^{*}=\{\mathrm{x} \in \mathrm{X} \mid \mathrm{U} \cap \mathrm{A} \notin \mathcal{I}$ for every $\mathrm{U} \in \tau(\mathrm{x})\}$. For $\mathrm{A} \subseteq(\mathrm{X}, \tau)$, it is known that $\mathrm{A}^{*}(\mathcal{N}(\tau), \tau)=\mathrm{Cl}(\operatorname{Int}(\mathrm{Cl}(\mathrm{A})))$ [5], and $\mathrm{A}^{*}(\mathcal{M}(\tau), \tau)$ is regular closed [5]. There is no known "closed form" for $\mathrm{A}^{*}(\mathcal{M}(\tau), \tau)$ For further details see [3].

A very useful fact about locally finite families is that they are closure preserving. The following theorem extends this result.

THEOREM II.3. Let $(X, \tau, \mathcal{I})$ be a space and let $\left\{A_{\alpha} \mid \alpha \in \Delta\right\}$ be a locally finite family of subsets of $\mathrm{X}$. Then

$$
\bigcup_{\alpha \in \Delta} A_{\alpha}^{*}=\left(\bigcup_{\alpha \in \Delta} A_{\alpha}\right)^{*}
$$

The simple proof is omitted.

In $T_{1}$ spaces, $A^{*}$ with respect to the ideal of finite sets is the derived set operator, usually denoted by $A^{\prime}$. Hence Theorem II. 3 shows that in $T_{1}$ spaces the derived set operator distributes across arbitrary unions of locally finite families. Since for $\mathrm{A} \subseteq(\mathrm{X}, \tau), \mathrm{Cl}(\mathrm{A})=\mathrm{A}^{*}(\{\emptyset\}, \tau)$, the well known closure preserving property of finite families is a corollary to the last theorem.

Given a space $(\mathrm{X}, \tau, \mathcal{I})$, we say $\mathcal{I}$ is $\underline{\tau \text {-boundary }}$ [8] or $\underline{\tau \text {-codense }}$ if $\mathcal{I} \cap \tau=\{\emptyset\}$, i.e. each member of $\mathcal{I}$ has empty $\tau$-interior In the next theorem we show that the class of almost paracompact spaces contains the class of $\mathcal{I}$-paracompact spaces when the ideal $\mathcal{I}$ is $\tau$-boundary.

THEOREM II.4. If $\mathcal{I}$ is $\tau$-boundary, and $(\mathrm{X}, \tau)$ is $\mathcal{I}$-paracompact then $(\mathrm{X}, \tau)$ is almost paracompact

PROOF. If $\mathcal{U}$ is any open cover of $\mathrm{X}$, let $\mathcal{V}$ be a locally finite open refinement of $\mathcal{U}$ such that $\mathrm{X}-\cup \mathcal{V} \in \mathcal{I}$. Since $\mathcal{I}$ is $\tau$-boundary, $\emptyset=\operatorname{Int}(\mathrm{A}-\cup \mathcal{V})=\mathbf{X}-\mathrm{Cl}(\mathbf{X}-(\mathbf{X}-\cup \mathcal{V}))=\mathbf{X}-\operatorname{Cl}(\cup \mathcal{V})$.

The following theorems examine the preservation of $\mathcal{I}$-paracompactness among the topologies $\tau, \tau^{*}$, and $\langle\psi(\tau)\rangle$, where this last topology is defined below.

THEOREM M.5. Let $(\mathrm{X}, \tau, \mathcal{I})$ be a space. If $\mathcal{I}$ is $\tau$-simple, $\tau$-boundary, and $\left(\mathrm{X}, \tau^{*}\right)$ is $\mathcal{I}$ paracompact, then $(\mathrm{X}, \tau)$ is $\mathcal{I}$-paracompact.

PROOF. Let $\mathcal{U}=\left\{\mathrm{U}_{\alpha} \mid \alpha \in \Delta\right\}$ be a $\tau$-open cover of $\mathrm{X}$ Then $\mathcal{U}$ is a $\tau^{*}$-open cover of $\mathrm{X}$ and hence has a $\tau^{*}$-locally finite $\tau^{*}$-open precise refinement $\left\{\mathrm{V}_{\alpha}-\mathrm{I}_{\alpha} \mid \mathrm{V}_{\alpha} \in \tau, \mathrm{I}_{\alpha} \in \mathcal{I}\right.$, and $\left.\alpha \in \Delta\right\}$ such that $\mathrm{X}-\mathrm{U}_{\alpha \in \Delta}\left(\mathrm{V}_{\alpha}-\mathrm{I}_{\alpha}\right)=\mathrm{J} \in \mathcal{I}$. Without loss of generality, assume $\mathrm{I}_{\alpha}=\mathrm{V}_{\alpha}-\mathrm{U}_{\alpha}$ so that $\mathrm{U}_{\alpha} \cap \mathrm{V}_{\alpha}$ $=\mathrm{V}_{\alpha}-\mathrm{I}_{\alpha}$. We claim that $\left\{\mathrm{V}_{\alpha} \mid \alpha \in \Delta\right\}$ is $\tau$-locally finite. Indeed, for $\mathrm{x} \in \mathrm{X}$, there exists $\mathrm{U}-\mathrm{I} \in \tau^{*}(\mathrm{x})(\mathrm{U} \in \tau(\mathrm{x}), \mathrm{I} \in \mathcal{I})$ such that $(\mathrm{U}-\mathrm{I}) \cap\left(\mathrm{V}_{\alpha}-\mathrm{I}_{\alpha}\right)=\emptyset$ for $\alpha \notin\left\{\alpha_{1}, \alpha_{2}, \ldots, \alpha_{\mathrm{n}}\right\}$. If $(\mathrm{U}-\mathrm{I}) \cap\left(\mathrm{V}_{\alpha}-\mathrm{I}_{\alpha}\right)=\emptyset$, then since $(\mathrm{U}-\mathrm{I}) \cap\left(\mathrm{V}_{\alpha}-\mathrm{I}_{\alpha}\right)=\left(\mathrm{U} \cap \mathrm{V}_{\alpha}\right)-\left(\mathrm{I} \cup \mathrm{I}_{\alpha}\right)$, we have 
$\left(U \cap V_{\alpha}\right)-\left(I \cup I_{\alpha}\right)=\emptyset$. This implies $U \cap V_{\alpha}=\emptyset$ since otherwise $U \cap V_{\alpha}$ is a nonempty $\tau$-open subset of $\mathrm{I} \cup \mathrm{I}_{\alpha}$ which contradicts the assumption that $\mathcal{I}$ is $\tau$-boundary If $\mathcal{V}=\left\{\mathrm{U}_{\alpha} \cap \mathrm{V}_{\alpha} \mid \alpha \in \Delta\right\}$, then $\mathcal{V}$ is $\tau$ locally finite since $\left\{\mathrm{V}_{\alpha} \mid \alpha \in \Delta\right\}$ is $\tau$-locally finite. Also, $\mathcal{V}$ is a $\tau$-open refinement of $\mathcal{U}$ and is an $\mathcal{I}$-cover of $\mathrm{X}$ since $\mathrm{X}-\cup_{\alpha \in \Delta}\left(\mathrm{U}_{\alpha} \cap \mathrm{V}_{\alpha}\right)=\mathrm{X}-\mathrm{U}_{\alpha \in \Delta}\left(\mathrm{V}_{\alpha}-\mathrm{I}_{\alpha}\right)=\mathrm{J}$.

If $(\mathrm{X}, \tau, \mathcal{I})$ is a space, we define a set operator $\psi: \mathcal{P}(\mathrm{X}) \rightarrow \tau$, where $\mathcal{P}(\mathrm{X})$ is the power set of $\mathrm{X}$, as follows [7]: if $\mathrm{A} \subseteq \mathrm{X}$, then $\psi(\mathrm{A})=\mathrm{X}-(\mathrm{X}-\mathrm{A})^{*}=U\{\mathrm{U} \in \tau \mid \mathrm{U}-\mathrm{A} \in \mathcal{I}\}$ Note that $\mathcal{I}$ is $\tau$-local if and only if $\psi(\mathrm{A})-\mathrm{A} \in \mathcal{I}$ for each $\mathrm{A} \subseteq \mathrm{X}$. If $\mathcal{B}$ is a basis for $\tau$, then $\psi(\mathcal{B})=\{\psi(\mathrm{B}) \mid \mathrm{B} \in \mathcal{B}\}$ is a basis for a topology coarser than $\tau$, denoted $\langle\psi(\mathcal{B})\rangle$. Furthermore, $\langle\psi(\mathcal{B})\rangle=\langle\psi(\tau)\rangle=\left\langle\psi\left(\tau^{*}\right)\right\rangle$ [7] Also, if $\mathcal{I}$ is $\tau$ local, $\langle\psi(\tau)\rangle=\langle\psi(\mathcal{P}(\mathrm{X}))\rangle$ since for $\mathrm{A} \subseteq \mathrm{X}, \psi(\mathrm{A})=\psi(\psi(\mathrm{A}))$

Let $(\mathrm{X}, \tau, \mathcal{I})$, be a space. We say that $\mathcal{I}$ is weakly $\tau$-local if $\mathrm{A}^{*}=\emptyset$ implies $\mathrm{A} \in \mathcal{I} \quad \mathcal{I}$ is called $\tau$ locally finite if the union of each $\tau$-locally finite family contained in $\mathcal{I}$ belongs to $\mathcal{I}$.

LEMMA II.6 [3]. Let (X, $\tau, \mathcal{I})$ be a space. Then $\mathcal{I}$ is $\tau$-local implies $\mathcal{I}$ is weakly $\tau$-local.

It is remarked in [3] that a space $(\mathrm{X}, \tau)$ is countably compact if and only if the ideal of finite sets, $I_{\mathrm{f}}$, is weakly $\tau$-local, whereas $\tau$-locality of $\mathcal{I}_{\mathrm{f}}$ is equivalent to hereditary compactness of $(\mathrm{X}, \tau)$. Therefore the implication in Lemma II. 6 is not reversible. The following example shows that an ideal can be $\tau$-locally finite and not weakly $\tau$-local.

EXAMPLE. Let $\mathrm{X}=[0, \Omega)$, where $\Omega$ denotes the first uncountable ordinal, and let $\tau$ denote the usual prder topology on $X$. Denote by $\mathcal{I}_{c}$ the ideal of countable subsets of $X$. Since $(X, \tau)$ is countably compact, any locally finite family of nonempty sets must be finite. Consequently, the union of any locally finite family contained in $\mathcal{I}_{c}$ belongs to $\mathcal{I}_{c}$, and hence $\mathcal{I}_{c}$ is $\tau$-locally finite. Since every point in $\mathrm{X}$ has a countable neighborhood, $\mathrm{A}^{*}=\emptyset$ for every $\mathrm{A} \subseteq \mathrm{X}$. In particular, $\mathrm{X}^{*}=\emptyset$ but $\mathrm{X} \notin \mathcal{I}_{\mathfrak{c}}$, and hence $\mathcal{I}_{\mathfrak{c}}$ is not weakly $\tau$-local.

THEOREM I.7. $\mathcal{I}$ is weakly $\tau$-local implies $\mathcal{I}$ is $\tau$-locally finite.

THEOREM II.8. If $(\mathrm{X}, \tau, \mathcal{I})$ is $\mathcal{I}$-paracompact, and $\mathcal{I}$ is $\tau$-locally finite, then $\mathcal{I}$ is weakly $\tau$-local

PROOF. Let $\mathrm{A}^{*}=\emptyset$. For every $\mathrm{x} \in \mathrm{X}$, there exists $U_{x} \in \tau(\mathrm{x})$ with $U_{\mathrm{x}} \cap \mathrm{A} \in \mathcal{I} \quad\left\{U_{\mathrm{x}} \mid \mathrm{x} \in \mathrm{X}\right\}$ is an open cover of $X$ and hence there exists a precise locally finite open refinement $\left\{V_{x} \mid x \in X\right\}$ which is an $I$ cover of $X$; i.e., $X-V=I \in I$ where $V=\cup_{x \in X} V_{x}$. Now $A=(A \cap V) \cup(A \cap I), A \cap I \in I$ and each $A \cap V_{x} \in \mathcal{I}$ by heredity. Thus, since $\left\{V_{x} \mid x \in X\right\}$ is $\tau$-locally finite, so is $\left\{A \cap V_{x} \mid x \in X\right\} \subseteq I$ Thus, $\cup_{x \in X}\left(A \cap V_{x}\right)=A \cap V \in \mathcal{I}$ since $\mathcal{I}$ is $\tau$-locally finite. So $A=(A \cap V) \cup(A \cap I) \in \mathcal{I}$. Thus, $\mathcal{I}$ is weakly $\tau$-local.

THEOREM II.9. If $(\mathrm{X}, \tau, \mathcal{I})$ is $\mathcal{I}$-paracompact and $\mathcal{I}$ is weakly $\tau$-local, then $\left(\mathrm{X}, \tau^{*}\right)$ is $\mathcal{I}$ paracompact.

PROOF. Every open cover can be refined by a basic open cover for which a locally finite refinement is a locally finite refinement of the original cover. So let $\mathcal{U}^{*}=\left\{\mathrm{U}_{\alpha}-\mathrm{I}_{\alpha} \mid \alpha \in \Delta, \mathrm{U}_{\alpha} \in \tau, \mathrm{I}_{\alpha} \in \mathcal{I}\right\}$ be a basic $\tau^{*}$-open cover of $\mathrm{X}$. Then $\mathcal{U}=\left\{\mathrm{U}_{\alpha} \mid \alpha \in \Delta\right\}$ is a $\tau$-open cover of $\mathrm{X}$ and has a $\tau$-locally finite $\tau$ open precise refinement $\mathcal{V}=\left\{\mathrm{V}_{\alpha} \mid \alpha \in \Delta\right\}$ which is an $\mathcal{I}$-cover of $\mathrm{X}$. Now $\mathcal{V}^{*}=\left\{\mathrm{V}_{\alpha}-\mathrm{I}_{\alpha} \mid \alpha \in \Delta\right\}$ is a $\tau$-locally finite $\tau^{*}$-open precise refinement of $\mathcal{U}^{*}$ and such that $\mathcal{V}^{*}$ is an $\mathcal{I}$-cover of $\mathrm{X}$ Now $\left\{\mathrm{V}_{\alpha} \cap \mathrm{I}_{\alpha} \mid \alpha \in \Delta\right\}$ is a $\tau$-locally finite subset of $\mathcal{I}$ and by weak $\tau$-locality of $\mathcal{I} ; \cup_{\alpha \in \Delta}\left(\mathrm{V}_{\alpha} \cap \mathrm{I}_{\alpha}\right) \in \mathcal{I}$. Let $\mathrm{X}-\cup \mathcal{V}=\mathrm{I} \in \mathcal{I}$, then $\mathrm{X}-\cup \mathcal{V}^{*} \subseteq \mathrm{I} \cup\left(\cup\left(\mathrm{V}_{\alpha} \cap \mathrm{I}_{\alpha}\right)\right) \in \mathcal{I}$. It remains only to show that $\mathcal{V}^{*}$ is $\tau^{*}$.

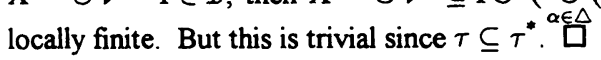

The following corollary is an immediate consequence of Theorems II.5 and II 9.

COROLLARY II.10. If $\mathcal{I}$ is $\tau$-local and $\tau$-boundary, then $(\mathrm{X}, \tau)$ is $\mathcal{I}$-paracompact if and only if $\left(\mathrm{X}, \tau^{*}\right)$ is $\mathcal{I}$-paracompact.

THEOREM II.11. If $\mathcal{I}$ is $\tau$-local, then $(\mathrm{X},<\psi(\tau)>)$ is $\mathcal{I}$-paracompact implies $(\mathrm{X}, \tau)$ is $\mathcal{I}$ paracompact. 
PROOF. Let $\mathcal{U}=\left\{\mathrm{U}_{\alpha} \mid \alpha \in \Delta\right\}$ be a $\tau$-open cover of $\mathrm{X}$. Then $\psi(\mathcal{U})=\left\{\psi\left(\mathrm{U}_{\alpha}\right) \mid \alpha \in \Delta\right\}$ is a $\langle\psi(\tau)\rangle$ open cover of $\mathrm{X}$ and has a $\langle\psi(\tau)\rangle$-locally finite $\langle\psi(\tau)\rangle$-open precise refinement $\mathcal{W}=\left\{\mathrm{W}_{\alpha} \mid \alpha \in \Delta\right\}$ which is an $\mathcal{I}$-cover of $\mathrm{X}$. Let $\mathcal{V}=\left\{\mathrm{W}_{\alpha} \cap \mathrm{U}_{\alpha} \mid \alpha \in \Delta\right\} . \mathcal{V}$ is a $\tau$-open (precise) refinement of $\mathcal{U}$ and since $\langle\psi(\tau)\rangle \subseteq \tau, \mathcal{W}$ is $\tau$-locally finite and so also $\mathcal{V}$ is $\tau$-locally finite By $\tau$-locality, $\mathrm{W}_{\alpha}-\left(\mathrm{W}_{\alpha} \cap \mathrm{U}_{\alpha}\right) \subseteq \psi\left(\mathrm{U}_{\alpha}\right)-\mathrm{U}_{\alpha} \in \mathcal{I}$ so that $\left\{\mathrm{W}_{\alpha}-\left(\mathrm{W}_{\alpha} \cap \mathrm{U}_{\alpha}\right) \mid \alpha \in \Delta\right\}$ is a $\tau$-locally finite subset of $\mathcal{I}$ and hence the union of this family is a member of $\mathcal{I}$. But, $\mathrm{X}-\mathrm{U}_{\alpha \in \Delta}\left(\mathrm{W}_{\alpha} \cap \mathrm{U}_{\alpha}\right) \subseteq$ $\left(\mathrm{X}-\cup_{\alpha \in \Delta} \mathrm{W}_{\alpha}\right) \cup\left(\cup_{\alpha \in \Delta}\left(\mathrm{W}_{\alpha}-\left(\mathrm{W}_{\alpha} \cap \mathrm{U}_{\alpha}\right)\right)\right) \in \mathcal{I}$ so that $\mathcal{V}$ is an $\mathcal{I}$-cover and $(\mathrm{X}, \tau)$ is $\mathcal{I}$-paracompact.

COROLLARY II.12. If $\mathcal{I}$ is $\tau$-local and $\tau$-boundary, then the following are equivalent.

(1) $(\mathrm{X},\langle\psi(\tau)\rangle)$ is $\mathcal{I}$-paracompact.

(2) $(X, \tau)$ is $\mathcal{I}$-paracompact.

(3) $\left(\mathrm{X}, \tau^{*}\right)$ is $\mathcal{I}$-paracompact

PROOF. (1) $\rightarrow$ (2) by Theorem II.11 and (2) is equivalent to (3) by Corollary II.10 To show (2) $\rightarrow$ (1), let $\mathcal{U}=\left\{\psi\left(\mathrm{U}_{\alpha}\right) \mid \alpha \in \triangle\right\}$ be a basic $\langle\psi(\tau)\rangle$-open cover of $\mathrm{X}$. Then $\mathcal{U}$ is a $\tau$-open cover of $\mathrm{X}$ and hence has a $\tau$-open $\tau$-locally finite precise refinement $\mathcal{V}=\left\{\mathrm{V}_{\alpha} \mid \alpha \in \Delta\right\}$ such that $\mathrm{X}-\cup \mathcal{V} \in \mathcal{I}$ Let $\psi(\mathcal{V})=\left\{\psi\left(\mathrm{V}_{\alpha}\right) \mid \alpha \in \Delta\right\}$. Each $\mathrm{V}_{\alpha} \subseteq \psi\left(\mathrm{U}_{\alpha}\right)$ hence $\psi\left(\mathrm{V}_{\alpha}\right) \subseteq \psi\left(\psi\left(\mathrm{U}_{\alpha}\right)\right)=\psi\left(\mathrm{U}_{\alpha}\right)$ (since $\left.\mathcal{I} \sim \tau\right)$, thus $\psi(\mathcal{V})$ is a $\langle\psi(\tau)\rangle$-open refinement of $\mathcal{U}$. Since $\mathrm{V}_{\alpha} \subseteq \psi\left(\mathrm{V}_{\alpha}\right)$ for every $\alpha$, we have $\mathrm{X}-\cup \psi(\mathcal{V}) \subseteq \mathrm{X}-\cup \mathcal{V} \in \mathcal{I}$; i.e., $\psi(\mathcal{V})$ is an $\mathcal{I}$-cover. To show that $\psi(\mathcal{V})$ is $\langle\psi(\tau)\rangle$-locally finite, let $\mathrm{x} \in \mathrm{X}$. There exists $\mathrm{U} \in \tau(\mathrm{x})$ such that $\mathrm{U} \cap \mathrm{V}_{\alpha}=\emptyset$ for $\alpha \notin\left\{\alpha_{1}, \alpha_{2}, \ldots, \alpha_{\mathrm{n}}\right\}$. We claim that $\mathrm{U} \cap \mathrm{V}_{\alpha}=\emptyset$ which implies $\mathrm{U} \cap \psi\left(\mathrm{V}_{\alpha}\right)=\emptyset$. Indeed, if $\mathrm{U} \cap \mathrm{V}_{\alpha}=\emptyset$ and $\mathrm{U} \cap \psi\left(\mathrm{V}_{\alpha}\right) \neq \emptyset$, then $\mathrm{U} \cap \psi\left(\mathrm{V}_{\alpha}\right) \subseteq \psi\left(\mathrm{V}_{\alpha}\right)-\mathrm{V}_{\alpha} \in \mathcal{I}$ (since $\mathcal{I} \sim \tau$ ), which contradicts the $\tau$-boundary assumption of $\mathcal{I}$.

The following is an example of an $\mathcal{I}$-paracompact space (actually paracompact) $(\mathrm{X}, \tau)$, such that $(\mathrm{X},\langle\psi(\tau)\rangle)$ is not $\mathcal{I}$-paracompact.

EXAMPLE. Let $X=R$ with $\tau$ the usual topology. Let $\mathcal{I}=\langle(0,3)\rangle=\{\mathrm{A} \subseteq \mathrm{X} \mid \mathrm{A} \subseteq(0,3)\}$ For every $\mathrm{U} \in \tau, \psi(\mathrm{U})=\mathrm{U} \cup(0,3)$ In particular, for any open set $\mathrm{G}$ in $\langle\psi(\tau)\rangle,(0,3) \subseteq \mathrm{G}$ Let $\mathcal{U}=\{(-\mathrm{n}, 3) \mid \mathrm{n} \in \mathrm{N}\} \cup\{(0, \mathrm{n}) \mid \mathrm{n} \in \mathrm{N}\}$, where $\mathrm{N}$ denotes the natural numbers, and observe that $\mathcal{U}$ is a $\langle\psi(\tau)\rangle$-open cover of $\mathrm{X}$ with the property that no finite open refinement of $\mathcal{U}$ can cover all of $\mathrm{X}$ with the exception of some subset of $(0,3)$. Also, no infinite open refinement of $\mathcal{U}$ can be locally finite since every open set in $\langle\psi(\tau)\rangle$ contains $(0,3)$. Thus, $(\mathrm{X},\langle\psi(\tau)\rangle)$ is not $\mathcal{I}$-paracompact. Since the ideal $\mathcal{I}$ is $\tau$-local but not $\tau$-boundary, we see that the $\tau$-boundary assumption cannot be omitted in Corollary II.12 for $(2) \rightarrow(1)$

Recall that if $(X, \tau)$ is a space, then $U \in \tau$ is called regular open if $U=\operatorname{Int}(\mathrm{Cl}(\mathrm{U}))$. The regular open subsets form a basis for a topology called the semiregularization of $\tau$, denoted $\tau_{\mathbf{s}}$. We remark that if $(\mathrm{X}, \tau, \mathcal{I})$ is a space with $\mathcal{I} \sim \tau$ and $\mathcal{N}(\tau) \subseteq \mathcal{I}$, then $\langle\psi(\tau)\rangle \subseteq \tau_{\mathrm{s}}$ [7]. If, in addition, $\mathcal{I}$ is $\tau$-boundary, then $\langle\psi(\tau)\rangle=\tau_{\mathrm{s}}$

COROLLARY II.13. Let $(\mathrm{X}, \tau, \mathcal{I})$ be a space with $\mathcal{I} \sim \tau, \mathcal{I} \tau$-boundary, and $\mathcal{N}(\tau) \subseteq \mathcal{I}$ Then $(\mathrm{X}, \tau)$ is $\mathcal{I}$ paracompact iff $\left(\mathrm{X}, \tau_{\mathrm{s}}\right)$ is $\mathcal{I}$-paracompact.

A space $(\mathrm{X}, \tau)$ is said to be semiregular if $\tau=\tau_{\mathrm{s}}$. A topological property is called semiregular if the property is always shared by a topology and its semiregularization. A property is called semi-topological if it is preserved by semi-homeomorphism in the sense of Crossley and Hildebrand [10] In [11], Hamlett and Rose show that the semi-topological properties are precisely the properties shared by $\tau$ and $\tau^{*}(\mathcal{N}(\tau))$ $\left(\tau^{*}(\mathcal{N}(\tau))\right.$ is denoted by $\tau^{\alpha}$ in the literature). Zahid observes in [1], that para-H-closedness is a semiregular property. Since $T_{2}$ is both a semiregular and semi-topological property, a stronger result follows. As a consequence, para-H-closedness is also a semi-topological property.

THEOREM II.14. Almost paracompactness (para-H-closedness) is a semiregular and semitopological property and for a space $(\mathrm{X}, \tau)$ the following are equivalent 
(1) $(\mathrm{X}, \tau)$ is almost paracompact

(2) $(\mathrm{X}, \tau)$ is $\mathcal{N}(\tau)$-paracompact.

(3) $\left(\mathrm{X}, \tau_{\mathrm{s}}\right)$ is $\mathcal{N}(\tau)$-paracompact.

(4) $\left(\mathrm{X}, \tau_{\mathrm{s}}\right)$ is $\mathcal{N}\left(\tau_{\mathrm{s}}\right)$-paracompact

(5) $\left(\mathrm{X}, \tau_{\mathrm{s}}\right)$ is almost paracompact.

(6) $\left(\mathrm{X}, \tau^{\alpha}\right)$ is $\mathcal{N}(\tau)$-paracompact.

(7) $\left(\mathrm{X}, \tau^{\alpha}\right)$ is almost paracompact

PROOF. For each $\mathrm{A} \subseteq \mathrm{X}$, since $\tau_{\mathrm{s}} \subseteq \tau, \mathrm{Cl}_{\tau} \mathrm{A} \subseteq \mathrm{Cl}_{\tau_{\mathrm{s}}} \mathrm{A}$ so that $\operatorname{Int}_{\tau_{\mathrm{s}}} \mathrm{Cl}_{\tau} \mathrm{A} \subseteq \operatorname{Int}_{\tau_{\mathrm{s}}} \mathrm{Cl}_{\tau_{\mathrm{s}}} \mathrm{A}$ But for each $\tau$-closed $\mathrm{F} \subseteq \mathrm{X}$, Int $\mathrm{In}_{\tau} \mathrm{F}=\operatorname{Int}_{\tau_{\mathrm{s}}} \mathrm{F}$. Thus, $\operatorname{Int}_{\tau_{\mathrm{s}}} \mathrm{Cl}_{\tau} \mathrm{A}=\operatorname{Int}_{\tau} \mathrm{Cl}_{\tau} \mathrm{A} \subseteq \operatorname{Int}_{\tau_{\mathrm{s}}} \mathrm{Cl}_{\tau_{\mathrm{s}}} \mathrm{A}$ for each $\mathrm{A} \subseteq \mathrm{X}$, and $\mathcal{N}\left(\tau_{\mathrm{s}}\right) \subseteq \mathcal{N}(\tau)$. Also, $\mathcal{N}(\tau) \cap \tau_{\mathrm{s}} \subseteq \mathcal{N}(\tau) \cap \tau=\{\emptyset\}$ implies that $\mathcal{N}(\tau)$ and $\mathcal{N}\left(\tau_{\mathrm{s}}\right)$ are each both $\tau$ boundary and $\tau_{\mathrm{s}}$-boundary. Now if $(\mathrm{X}, \tau)$ is almost paracompact, $(\mathrm{X}, \tau)$ is $\mathcal{N}(\tau)$-paracompact by Theorem II. 1 (1), so that by Corollary II.12, (X, $\left.\tau_{\mathrm{s}}\right)$ is $\mathcal{N}(\tau)$-paracompact. By Theorem II 4, since $\mathcal{N}(\tau)$ is $\tau_{\mathrm{s}}$-boundary, $\left(\mathrm{X}, \tau_{\mathrm{s}}\right)$ is almost paracompact, and therefore by Theorem II.1 (1), (X, $\left.\tau_{\mathrm{s}}\right)$ is $\mathcal{N}\left(\tau_{\mathrm{s}}\right)$ paracompact.

Conversely, if $\left(\mathrm{X}, \tau_{\mathrm{s}}\right)$ is almost paracompact and therefore $\mathcal{N}\left(\tau_{\mathrm{s}}\right)$-paracompact, then since $\mathcal{N}\left(\tau_{\mathrm{s}}\right) \subseteq \mathcal{N}(\tau)$, by Theorem II.2, (X, $\left.\tau_{\mathrm{s}}\right)$ is $\mathcal{N}(\tau)$-paracompact. Then by Corollary II. 13, (X, $\left.\tau\right)$ is $\mathcal{N}(\tau)$ paracompact and hence $(\mathrm{X}, \tau)$ is almost paracompact.

Since $\mathcal{N}(\tau)$ is $\tau$-local and $\tau$-boundary and since $\mathcal{N}\left(\tau^{\alpha}\right)=\mathcal{N}\left(\tau^{*}(\mathcal{N}(\tau))=\mathcal{N}(\tau)\right.$, by Corollary II 10, $(\mathrm{X}, \tau)$ is almost paracompact iff $\left(\mathrm{X}, \tau^{\alpha}\right)$ is almost paracompact. So almost paracompactness is a semitopological property Since the $T_{2}$ axiom is both a semiregular and semi-topological property, so is paraH-closedness

A collection $\mathcal{A}$ of subsets of a space $(\mathrm{X}, \tau)$ is said to be $\sigma$-locally finite if $\mathcal{A}=\bigcup_{\mathrm{n}=1}^{\infty} \mathcal{A}_{\mathrm{n}}$ where each $\mathcal{A}_{\mathrm{n}}$ is a locally finite family Zahid [1] shows that a $T_{2}$ space is para-H-closed iff every open cover $\mathcal{U}$ of the space has a $\sigma$-locally finite refinement $\mathcal{V}=\bigcup_{\mathrm{n}=1}^{\infty} \mathcal{V}_{\mathrm{n}}$ such that $\mathrm{X}=\bigcup_{\mathrm{n}=1}^{\infty} \operatorname{Int}\left(\operatorname{Cl}\left(\cup \mathcal{V}_{\mathrm{n}}\right)\right)$. This result is generalized in the following theorem.

THEOREM II.15. Let $(\mathrm{X}, \tau, \mathcal{I})$ be a space with $\mathcal{N}(\tau) \subseteq \mathcal{I}$, and $\mathcal{I} \tau$-boundary. Then $(\mathrm{X}, \tau, \mathcal{I})$ is $\mathcal{I}$ paracompact iff every open cover $\mathcal{U}$ of $\mathrm{X}$ has a $\sigma$-locally finite refinement $\mathcal{V}=\bigcup_{\mathrm{n}=1}^{\infty} \mathrm{V}_{\mathrm{n}}$ such that $\mathrm{X}=\bigcup_{\mathrm{n}=1}^{\infty}$ Int $\mathrm{Cl}\left(\cup \mathcal{V}_{\mathrm{n}}\right)$

PROOF. Necessity is obvious. To show sufficiency, let $\mathcal{U}$ be an open cover of $\mathrm{X}$ and suppose $\mathcal{U}$ has a $\sigma$-locally finite refinement $\mathcal{V}=\bigcup_{\mathrm{n}=1}^{\infty} \mathcal{V}_{\mathrm{n}}$ such that $\mathrm{X}=\bigcup_{\mathrm{n}=1}^{\infty} \operatorname{Int} \mathrm{Cl}\left(\cup \mathcal{V}_{\mathrm{n}}\right)$. Let $\mathrm{O}_{\mathrm{n}}=\cup \mathcal{V}_{\mathrm{n}}$ so that $\mathrm{X}=$ $\bigcup_{n=1}^{\infty}$ Int $\mathrm{Cl}\left(O_{n}\right)$. Let $P_{1}=O_{1}$, and $P_{n}=O_{n}-\left(\bigcup_{n=1}^{n-1} O_{i}\right)^{*}$ for $n>1$. Let $\xi_{n}=\left\{V \cap P P_{n} \mid V \in \mathcal{V}_{n}\right\}$ for each $n=$ $1,2,3, \ldots$, and let $\xi=\bigcup_{n=1}^{\infty} \xi_{n}$ Observe that $\xi$ is an open refinement of $\mathcal{V}$ and hence $\mathcal{U}$. We claim that $\xi$ is a locally finite family. Indeed, let $\mathrm{x} \in \mathrm{X}$, and let $\mathrm{n}_{\mathrm{x}}=\min \left\{\mathrm{n}: \mathrm{x} \in \operatorname{Int} \mathrm{Cl}\left(\mathrm{O}_{\mathrm{n}}\right)\right\}$ Then $\mathrm{x} \in \operatorname{Int} \mathrm{Cl}\left(\mathrm{O}_{\mathrm{n}_{\mathrm{x}}}\right)$ and Int $\mathrm{Cl}\left(\mathrm{O}_{\mathrm{n}_{x}}\right) \cap \mathrm{P}_{\mathrm{n}}=\emptyset$ for every $\mathrm{n}>\mathrm{n}_{\mathrm{x}}$ i.e., $\mathrm{P}_{\mathrm{n}}=\mathrm{O}_{\mathrm{n}}-\left(\mathrm{i}<\mathrm{n}_{\mathrm{O}}\right)^{*}$ and Int $\mathrm{Cl}\left(\mathrm{O}_{\mathrm{n}_{x}}\right) \subseteq \mathrm{O}_{\mathrm{n}_{\mathrm{x}}}^{*}$ [7] Thus (Int $\left.\mathrm{Cl}\left(\mathrm{O}_{\mathrm{n}_{\mathbf{x}}}\right)\right) \cap\left(\cup \xi_{\mathrm{n}}\right)=\emptyset$ for every $\mathrm{n}>\mathrm{n}_{\mathbf{x}}$. For each $\mathrm{n}=1,2, \ldots, \mathrm{n}_{\mathbf{x}}$, $\mathrm{x}$ has a neighborhood $\mathrm{G}_{\mathrm{n}} \in \tau(\mathrm{x})$ such that $G_{n}$ intersects at most finitely many members of $\xi_{n}$ Thus (Int $\left.C l\left(O_{n_{x}}\right)\right) \cap G_{1} \cap \ldots \cap G_{n_{x}}$ is a neighborhood of $\mathrm{x}$ which intersects at most finitely many members of $\xi$

We conclude the proof by showing that $X-\cup \xi \in \mathcal{N}(\tau)$ We proceed by showing (1) $X=\bigcup_{n=1}^{\infty} P_{n}^{*}$, and (2) $\mathrm{X} \subseteq(\cup \xi)^{*}$. The result then follows from the fact that $(\cup \xi)^{*}-(\cup \xi) \subseteq \mathrm{Cl}(\cup \xi)-(\cup \xi) \in \mathcal{N}(\tau)$

(1) By assumption, $\mathrm{X}=\bigcup_{\mathrm{n}=1}^{\infty} \operatorname{Int} \mathrm{Cl}\left(\mathrm{O}_{\mathrm{n}}\right)$, and Int $\mathrm{Cl}\left(\mathrm{O}_{\mathrm{n}}\right) \subseteq \mathrm{O}_{\mathrm{n}}^{*}$ since $\mathcal{I}$ is $\tau$-boundary Let $\mathrm{x} \in \mathrm{X}$ and let $\mathrm{m}_{\mathrm{x}}=\min \left\{\mathrm{n} \mid \mathrm{x} \in \mathrm{O}_{\mathrm{n}}^{*}\right\}$, then $\mathrm{x} \in \mathrm{O}_{\mathrm{m}_{\mathrm{x}}}^{*}-\left(\mathrm{U}_{1<\mathrm{m}_{\mathrm{x}}} \mathrm{O}_{1}^{*}\right) \subseteq \mathrm{P}_{\mathrm{m}_{\mathrm{x}}}^{*}$ Thus $\mathrm{X} \subseteq \bigcup_{\mathrm{n}=1}^{\infty} \mathrm{P}_{\mathrm{n}}^{*}$ 
(2)

$$
\begin{aligned}
(\cup \xi)^{*} & =\left[\cup\left(\bigcup_{n=1}^{\infty} \xi_{n}\right)\right]^{*} \\
& =\left[\bigcup_{n=1}^{\infty}\left(\cup \xi_{n}\right)\right]^{*} \\
& \supseteq \bigcup_{n=1}^{\infty}\left[\cup \xi_{n}\right]^{*} \\
& =\bigcup_{n=1}^{\infty}\left[\bigcup_{V \in \nu_{n}}\left(V \cap P_{n}\right)\right]^{*} \\
& =\bigcup_{n=1}^{\infty}\left[P_{n} \cap\left(\cup_{V \in \nu_{n}} V\right)\right]^{*} \\
& =\bigcup_{n=1}^{\infty}\left[P_{n} \cap O_{n}\right]^{*} \\
& =\bigcup_{n=1}^{\infty} P_{n}^{*} \\
& =X \square
\end{aligned}
$$

Recall that a space $(X, \tau)$ is a Baire space iff $\mathcal{M}(\tau) \cap \mathcal{I}=\{\emptyset\}$; i.e., $\mathcal{M}(\tau)$ is $\tau$-boundary.

COROLLARY II.16. Let $(\mathrm{X}, \tau, \mathcal{I})$ be a space with $\mathcal{I} \tau$-boundary and $\mathcal{N}(\tau) \subseteq \mathcal{I}$. Then $(\mathrm{X}, \tau)$ is $\mathcal{I}$ paracompact iff $(X, \tau)$ is almost paracompact In particular, if $(X, \tau)$ is a Baire space, then $(\mathrm{X}, \tau)$ is $\mathcal{M}(\tau)$ paracompact iff $(X, \tau)$ is almost paracompact.

PROOF. Theorem II. 15 provides a common equivalent condition for $(\mathrm{X}, \tau)$ to be $\mathcal{I}$-paracompact

In semi-regular spaces, $\mathcal{I}$-paracompactness with respect to a $\tau$-boundary ideal can be characterized as follows.

THEOREM I.17. Let $(\mathrm{X}, \tau, \mathcal{I})$ be semiregular with $\mathcal{I} \tau$-boundary. Then $(\mathrm{X}, \tau)$ is $\mathcal{I}$-paracompact iff every regular open cover $\mathcal{U}$ of $\mathrm{X}$ has a locally finite refinement $\mathcal{A}$ (not necessarily open) such that $\mathrm{X}-\cup \mathcal{A} \in \mathcal{I}$.

PROOF. Necessity is obvious. To show sufficiency, let $\mathcal{U}=\left\{\mathrm{U}_{\alpha} \mid \alpha \in \triangle\right\}$ be a regular open cover of $\mathrm{X}$ and assume $\mathcal{A}=\left\{\mathrm{A}_{\alpha} \mid \alpha \in \triangle\right\}$ is a precise locally finite refinement of $\mathcal{U}$ such that $\mathrm{X}-\cup \mathcal{A} \in \mathcal{I}$. For each $\alpha \in \Delta$, we have $\mathrm{A}_{\alpha} \subseteq \mathrm{U}_{\alpha}$ and hence $\psi\left(\mathrm{U}_{\alpha}\right)=\mathrm{U}_{\alpha}[7$, Theorem 5, (5)]. Now $\mathcal{V}=$ $\left\{\psi\left(\mathrm{A}_{\alpha}\right) \mid \alpha \in \triangle\right\}$ is an open refinement of $\mathcal{U}$ and $\mathrm{X}-\cup \mathcal{V} \subseteq \mathrm{X}-\cup \mathcal{A} \in \mathcal{I}$ To show $\mathcal{V}$ is locally finite, let $\mathrm{x} \in \mathrm{X}$ There exists $\mathrm{U} \in \tau(\mathrm{x})$ such that $\mathrm{U} \cap \mathrm{A}_{\alpha}=\emptyset$ for $\alpha \notin\left\{\alpha_{1}, \alpha_{2}, \ldots, \alpha_{\mathrm{n}}\right\}$ Observe that $\mathrm{U} \cap \mathrm{A}_{\alpha}=\emptyset$ which implies $U \cap \psi\left(A_{\alpha}\right)=\emptyset$; i.e., if $\mathrm{y} \in U$ and $V \in \tau(y)$, then $V-A_{\alpha} \supseteq V \cap U \notin I$ so that $y \notin \psi\left(A_{\alpha}\right)$ Thus $\mathrm{U} \cap \psi\left(\mathrm{A}_{\alpha}\right)=\emptyset$ for $\alpha \notin\left\{\alpha_{1}, \alpha_{2}, \ldots, \alpha_{\mathrm{n}}\right\}$

The following corollary applies the previous theorem to the ideal of nowhere dense sets.

THEOREM II.18. Let $(\mathrm{X}, \tau)$ be a (Hausdorff) space. Then $(\mathrm{X}, \tau)$ is almost paracompact (para-Hclosed) iff every regular open cover of $\mathrm{X}$ has a locally finite refinement, not necessarily open, whose union is dense in $X$.

PROOF. The necessity is clear since a cover of an almost paracompact (para-H-closed) space by regular open sets is an open cover and since locally finite families are closure preserving For the sufficiency, by Theorem II 14 it is enough to show that $\left(\mathrm{X}, \tau_{\mathbf{s}}\right)$ is $\mathcal{N}(\tau)$-paracompact. But by hypothesis, every regular open cover $\mathcal{U}$ of $\mathrm{X}$ has a $\tau$-locally finite refinement $\mathcal{A}$ such that $\mathrm{X}-\cup \mathcal{A} \in \mathcal{N}(\tau)$ Since $\tau_{\mathrm{s}} \subseteq \tau, \mathcal{U}$ is locally finite with respect to $\tau_{\mathrm{s}}$ and since $\left(\mathrm{X}, \tau_{\mathrm{s}}\right)$ is semiregular, by Theorem II. 17, $\left(\mathrm{X}, \tau_{\mathrm{s}}\right)$ is $\mathcal{N}(\tau)$-paracompact

\section{PRESERVATION BY FUNCTIONS AND PRODUCTS}

It was shown by Michael in [14] that the closed continuous image of a paracompact (Hausdorff) space is paracompact and Zahid has shown [1] that a perfect (continuous, closed, compact fibers) image of a para-H-closed space is para-H-closed in the category of Hausdorff spaces. In this more general setting we offer the following result. First, for any function $f: X \rightarrow Y$ and subset $A \subseteq X$, let $f^{\#}(A)=\{y \in$ $\left.Y \mid f^{-1}(y) \subseteq A\right\}=Y-f(X-A)$. Then $f$ is closed iff $f^{\sharp}(U)$ is open for each open subset $U$ of $X$ 
THEOREM III.1. Let $\mathrm{f}:(\mathrm{X}, \tau, \mathcal{I}) \rightarrow(\mathrm{Y}, \sigma, \mathcal{J})$ be a continuous open closed surjection with $\mathrm{f}^{-1}(\mathrm{y})$ compact for every $\mathrm{y} \in \mathrm{Y}$ and $\mathrm{f}(\mathcal{I}) \subseteq \mathcal{J}$. If $(\mathrm{X}, \tau, \mathcal{I})$ is $\mathcal{I}$-paracompact, then $(\mathrm{Y}, \sigma, \mathcal{J})$ is $\mathcal{J}$-paracompact.

PROOF. Let $\left\{\mathrm{U}_{\alpha} \mid \alpha \in \Delta\right\}$ be an open cover of $\mathrm{Y}$. Then $\left\{\mathrm{f}^{-1}\left(\mathrm{U}_{\alpha}\right) \mid \alpha \in \Delta\right\}$ is an open cover of $\mathrm{X}$ and hence there exists a locally finite precise refinement $\left\{\mathrm{V}_{\alpha} \mid \alpha \in \Delta\right\}$ of $\left\{\mathrm{f}^{-1}\left(\mathrm{U}_{\alpha}\right) \mid \alpha \in \Delta\right\}$ such that $\mathrm{X}-\underset{\alpha \in \Delta}{\cup} \mathrm{V}_{\alpha}=\mathrm{I} \in I$. Now $\left\{f\left(\mathrm{~V}_{\alpha}\right) \mid \alpha \in \Delta\right\}$ is a precise open refinement of $\left\{\mathrm{U}_{\alpha} \mid \alpha \in \Delta\right\}$ and $\mathrm{Y}=$ $f(X)=f\left(\left(\bigcup_{\alpha \in \Delta} V_{\alpha}\right) \cup I\right)=\bigcup_{\alpha \in \Delta} f\left(V_{\alpha}\right) \cup f(I)$ so that $Y-\bigcup_{\alpha \in \Delta} f\left(V_{\alpha}\right) \subseteq f(I) \in \mathcal{J}$. To show that $\left\{\mathrm{f}\left(\mathrm{V}_{\alpha}\right) \mid \alpha \in \Delta\right\}$ is locally finite, let $\mathrm{y} \in \mathrm{Y}$; then there exists an open set $\mathrm{O}$ such that $\mathrm{f}^{-1}(\mathrm{y}) \subseteq \mathrm{O}$ and $O \cap \mathrm{V}_{\alpha}=\emptyset$ for $\alpha \notin\left\{\alpha_{1}, \alpha_{2}, \ldots, \alpha_{\mathrm{n}}\right\}$. Now $\mathrm{f}^{\sharp}(\mathrm{O}) \cap \mathrm{f}\left(\mathrm{V}_{\alpha}\right) \neq \emptyset$ implies $\mathrm{O} \cap \mathrm{V}_{\alpha} \neq \emptyset$ Hence $\mathrm{f}^{\sharp}(0)$ is an open neighborhood of $y$ which intersects at most finitely many sets from the collection $\left\{f\left(V_{\alpha}\right) \mid \alpha \in \triangle\right\}$

The theorems of Michael and Zahid mentioned above are sharper in their special case settings than what the previous theorem provides. The previous theorem though does lead to some meaningful consequences.

COROLLARY III.2. Let $\mathrm{f}$. $(\mathrm{X}, \tau, \mathcal{I}) \rightarrow(\mathrm{Y}, \sigma, \mathcal{J})$ be a homeomorphism with $\mathrm{f}(\mathcal{I}) \subseteq \mathcal{J}$ If $(\mathrm{X}, \tau)$ is $\mathcal{I}$-paracompact then $(\mathrm{Y}, \sigma)$ is $\mathcal{J}$-paracompact.

In the language of [11], $\mathcal{I}$-paracompact is a "*-topological" property.

We will say that a function $\mathrm{f}:(\mathrm{X}, \tau . \mathcal{I}) \rightarrow(\mathrm{Y}, \sigma, \mathcal{J})$ is $\psi$-continuous iff $\mathrm{f}:(\mathrm{X}, \tau, \mathcal{I}) \rightarrow(\mathrm{Y},\langle\psi(\sigma)\rangle)$ is continuous. Certainly every continuous function is $\psi$-continuous since $\langle\psi(\sigma)\rangle \subseteq \sigma$ and the converse is not true. We remark that the almost continuous functions of Singal and Singal [15] are a special case where $\mathcal{J}$ is the nowhere dense ideal on the space $(Y, \sigma)$.

We remark that it is clear from the proof of Zahid's result [1] (that perfect images of para-H-closed spaces are para-H-closed in the category of Hausdorff spaces) that it is sufficient for the function to be almost continuous ( $\psi$-continuous with respect to the ideal of nowhere dense sets on the co-domain)

It is well known that perfect preimages of paracompact spaces are paracompact [16] and Zahid [1] shows that perfect preimages of para-H-closed spaces are para-H-closed in the category of Hausdorff spaces. We remark that his proof shows that perfect preimages of almost paracompact spaces are almost paracompact. In this spirit we have the following result. Given a function $\mathrm{f:}(\mathrm{X}, \tau) \rightarrow(\mathrm{Y}, \sigma, \mathcal{J})$, we denote by $\left\langle\mathrm{f}^{-1}(\mathcal{J})\right\rangle$ the ideal generated by preimages of members of $\mathcal{J}$, i.e. $\left\langle\mathrm{f}^{-1}(\mathcal{J})\right\rangle=\left\{\mathrm{A} \mid \mathrm{A} \subseteq \mathrm{f}^{-1}(\mathrm{~J})\right.$ for some $\mathrm{J} \in \mathcal{J}\}$

THEOREM III.3. Let $\mathrm{f}:(\mathrm{X}, \tau, \mathcal{J}) \rightarrow(\mathrm{Y}, \sigma, \mathcal{J})$ be a perfect function from a space $\mathrm{X}$ onto a $\mathcal{J}$ paracompact space $\mathrm{Y}$, with $\left\langle\mathrm{f}^{-1}(\mathcal{J})\right\rangle \subseteq \mathcal{I}$. then $(\mathrm{X}, \tau)$ is $\mathcal{I}$-paracompact.

PROOF. Let $\mathcal{U}=\left\{\mathrm{U}_{\sigma} \mid \sigma \in \triangle\right\}$ be an open cover of $\mathrm{X}$. Let $\mathcal{F}=\{\mathrm{F} \subseteq \triangle \mid \mathrm{F}$ is finite $\}$ and let $\mathrm{U}_{\mathrm{F}}=$ $\cup_{\alpha \in \mathrm{F}} \mathrm{U}_{\alpha}$ for $\mathrm{F} \in \mathcal{F}$. Let $\mathcal{U}^{\prime}=\left\{\mathrm{U}_{\mathrm{F}} \mid \mathrm{F} \in \mathcal{F}\right\}$ Observe that $\left\{\mathrm{f}^{\sharp}\left(\mathrm{U}_{\mathrm{F}}\right) \mid \mathrm{F} \in \mathcal{F}\right\}$ is an open cover of $\mathrm{Y}$

Indeed, if $y \in Y$ then $\mathrm{f}^{-1}(\mathrm{y})$ is compact implies there exists a finite subcollection $\left\{\mathrm{U}_{\alpha_{1}}, \ldots, \mathrm{U}_{\alpha_{\mathrm{n}}}\right\}$ such that $\mathrm{f}^{-1}(\mathrm{y}) \subseteq \bigcup_{\mathrm{i}=1}^{\mathrm{n}} \mathrm{U}_{\alpha_{1}}$. Letting $\mathrm{F}=\left\{\alpha_{1}, \ldots, \alpha_{\mathrm{n}}\right\}$, we have $\mathrm{y} \in \mathrm{f}^{\#}\left(\mathrm{U}_{\mathrm{F}}\right)$ Now, since $(\mathrm{Y}, \sigma)$ is $\mathcal{J}$-paracompact, there exists a precise open locally finite refinement $\left\{\mathrm{V}_{\mathrm{F}} \mid \mathrm{F} \in \mathcal{F}\right\}$ of $\left\{\mathrm{f}^{\sharp}\left(\mathrm{U}_{\mathrm{F}}\right) \mid \mathrm{F} \in \mathcal{F}\right\}$ such that $\mathrm{Y}=(\underset{\mathrm{F} \in \mathcal{F}}{U}$ $\left.V_{F}\right) \cup J$ for some $J \in \mathcal{J}$. Let $\mathcal{V}=\left\{\mathrm{f}^{-1}\left(V_{\mathrm{F}}\right) \cap \mathrm{U}_{\alpha} \mid \mathrm{F} \in \mathcal{F}\right.$ and $\left.\alpha \in \mathrm{F}\right\}$. Then $\mathcal{V}$ is an open refinement of $\mathcal{U}$ and we claim: (1) $\mathcal{V}$ is locally finite, and (2) $\mathcal{V}$ is an $\mathcal{I}$-cover of $\mathrm{X}$ To show (1), let $\mathrm{x} \in \mathrm{X}$ Then there exists $\mathrm{V} \in \sigma(\mathrm{f}(\mathrm{x}))$ such that $\mathrm{V} \cap \mathrm{V}_{\mathrm{F}} \neq \emptyset$ for finitely many members $\mathrm{F}$ of $\mathcal{F}$. Now observe that $\mathrm{f}^{-1}(\mathrm{~V}) \cap \mathrm{f}^{-1}\left(\mathrm{~V}_{\mathrm{F}}\right) \neq \emptyset$ iff $\mathrm{V} \cap \mathrm{V}_{\mathrm{F}} \neq \emptyset$ showing that $\mathrm{f}^{-1}(\mathrm{~V})$ intersects at most finitely many members of $\mathcal{V}$. To show (2), observe that for every $\mathrm{F} \in \mathcal{F}, \mathrm{f}^{-1}\left(\mathrm{~V}_{\mathrm{F}}\right)=\mathrm{U}_{\mathrm{F}}-\mathrm{I}_{\mathrm{F}}$ where $\mathrm{I}_{\mathrm{F}} \subseteq \mathrm{f}^{-1}(\mathrm{~J})$. Now for $\mathrm{F} \in \mathcal{F}$ and $\alpha \in \mathrm{F}$, we have $\mathrm{f}^{-1}\left(\mathrm{~V}_{\mathrm{F}}\right) \cap \mathrm{U}_{\alpha}=\left(\mathrm{U}_{\mathrm{F}}-\mathrm{I}_{\mathrm{F}}\right) \cap \mathrm{U}_{\alpha}=\mathrm{U}_{\alpha}-\mathrm{I}_{\mathrm{F}}$. Hence $\mathrm{X}-U \mathcal{V}=\mathrm{X}-\cup\left\{\mathrm{U}_{\alpha}-\mathrm{I}_{\mathrm{F}} \mid \mathrm{F} \in \mathcal{F}\right.$ and $\alpha \in \mathrm{F}\} \subseteq \cup\left\{\mathrm{I}_{\mathrm{F}} \mid \mathrm{F} \in \mathcal{F}\right\} \subseteq \mathrm{f}^{-1}(\mathrm{~J})$.

It is well known that the product of two paracompact spaces is not necessarily paracompact However, it was shown by Dieudonné in [17] that the product of a paracompact space with a compact 
space is paracompact. Zahid shows in [1] that the product of a para-H-closed space and an H-closed space [18] is para-H-closed. In this spirit, we offer the following result.

COROLLARY II.4. Let $(\mathrm{X}, \tau, \mathcal{I})$ be an $\mathcal{I}$-paracompact Hausdorff space, let $(\mathrm{Y}, \sigma)$ be a compact space, and let $\mathrm{p}: \mathrm{X} \times \mathrm{Y} \rightarrow \mathrm{X}$ be the projection function. If $\mathcal{J}$ is an ideal on $\mathrm{X} \times \mathrm{Y}$ such that $\left\langle\mathrm{p}^{-1}(\mathcal{I})\right\rangle \subseteq \mathcal{J}$, then $\mathrm{X} \times \mathrm{Y}$ is $\mathcal{J}$-paracompact.

PROOF. The projection function $\mathrm{p} \cdot \mathrm{X} \times \mathrm{Y}$ is perfect. The result follows immediately then from Theorem III. 3

\section{SUBSETS}

If $\mathcal{I}$ is an ideal on a nonempty space $(\mathrm{X}, \tau)$ and $\mathrm{A} \subseteq \mathrm{X}$, we denote the restriction of $\mathcal{I}$ to $\mathrm{A}$ by $\mathcal{I} \mid \mathrm{A}=$ $\{\mathrm{I} \cap \mathrm{A} \mid \mathrm{I} \in \mathcal{I}\}=\{\mathrm{B} \subseteq \mathrm{A} \mid \mathrm{B} \in \mathcal{I}\}$. We say that $\mathrm{A}$ is an $\mathcal{I}$-paracompact subset if for every open cover $\mathcal{U}$ of A there exists a locally finite (with respect to $\tau$ ) open refinement $\mathcal{V}$ of $\mathcal{U}$ such that $\mathrm{A}-\cup \mathcal{V} \in \mathcal{I}$. If $\mathcal{I}=$ $\{\emptyset\}$, then the definition of $A$ being a " $\{\emptyset\}$-paracompact subset" coincides with the definition of $A$ being an " $\alpha$-paracompact" subset in [19]. We will say $A$ is an $I$-paracompact subspace if $(X, \tau|A, I| A)$ is $I$ paracompact as a subspace, where $\tau \mid \mathrm{A}$ is the usual subspace topology. The definition of $\mathrm{A}$ being a " $\{\emptyset\}$ paracompact subspace" coincides with A being a " $\beta$-paracompact" subset in [19].

THEOREM IV.1. If $A \subseteq(\mathrm{X}, \tau, \mathcal{I})$ is an $\mathcal{I}$-paracompact subset, then $\mathrm{A}$ is an $\mathcal{I}$-paracompact subspace

PROOF. Let $\mathcal{U}=\left\{\mathrm{U}_{\alpha} \cap \mathrm{A} \mid \alpha \in \triangle\right\}$ be a $\tau \mid \mathrm{A}$-open cover of $\mathrm{A}$ where $\mathrm{U}_{\alpha} \in \tau$ for each $\alpha \in \Delta$. Then $\left\{U_{\alpha} \mid \alpha \in \Delta\right\}$ is a $\tau$-open cover of $A$ and hence has a $\tau$-open $\tau$-locally finite precise refinement $\left\{\mathrm{V}_{\alpha} \mid \alpha \in \Delta\right\}$ such that $\mathrm{A}-\cup\left\{\mathrm{V}_{\alpha} \mid \alpha \in \Delta\right\} \in \mathcal{I}$. Now $\mathcal{V}=\left\{\mathrm{V}_{\alpha} \cap \mathrm{A} \mid \alpha \in \Delta\right\}$ is a $\tau \mid \mathrm{A}$-open $\tau \mid \mathrm{A}$ locally finite refinement of $\mathcal{U}$ and $\mathrm{A}-U \mathcal{V}=\mathrm{A}-U\left\{\mathrm{~V}_{\alpha} \mid \alpha \in \Delta\right\} \in \mathcal{I}$.

The converse of the above theorem is false as shown by an example of an $\{\emptyset\}$-paracompact subspace ( $\beta$-paracompact subset) which is not an $\{\emptyset\}$-paracompact subset ( $\alpha$-paracompact subset) in [19].

Zahid defines a subset $\mathrm{A}$ of a Hausdorff space $(\mathrm{X}, \tau)$ to be para-H-closed if it is para-H-closed as a subspace; i.e., if $(\mathrm{A}, \tau \mid \mathrm{A})$ is para-H-closed and hence if $(\mathrm{A}, \tau \mid \mathrm{A})$ is $\mathcal{N}(\tau \mid \mathrm{A})$-paracompact. Observe that $\mathcal{N}(\tau \mid \mathrm{A}) \subseteq \mathcal{N}(\tau) \mid \mathrm{A}$ but the reverse inclusion may not hold. It is shown in [20], however that $\mathcal{N}(\tau) \mid \mathrm{A} \subseteq \mathcal{N}(\tau \mid \mathrm{A})$, and hence $\mathcal{N}(\tau) \mid \mathrm{A}=\mathcal{N}(\tau \mid \mathrm{A})$ if $\mathrm{A} \subseteq \mathrm{Cl}(\operatorname{Int}(\mathrm{Cl}(\mathrm{A})))$. Thus we have the following theorem.

THEOREM IV.2. If $A \subseteq(X, \tau)$ is a para-H-closed subspace, then $(\mathrm{A}, \tau \mid \mathrm{A})$ is a $\mathcal{N}(\tau)$-paracompact subspace. The converse is true if $\mathrm{A} \subseteq \mathrm{Cl}(\operatorname{Int}(\mathrm{Cl}(\mathrm{A})))$

We have the following diagram:

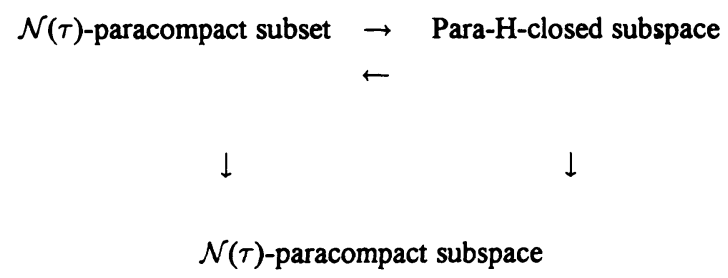

Figure 1

Paracompactness is well known to be closed hereditary (in fact $\mathrm{F}_{\sigma}$ subsets of paracompact Hausdorff spaces are paracompact as subspaces), but Zahid [1] provides an example which shows that even Hclosed spaces may have closed subsets which are not para-H-closed subspaces

THEOREM IV.3. Let $(\mathrm{X}, \tau, \mathcal{I})$ be an $\mathcal{I}$-paracompact space. If $\mathrm{A} \subseteq \mathrm{X}$ is closed, then $\mathrm{A}$ is an $\mathcal{I}$ paracompact subset. 
PROOF. Let $\mathcal{U}=\left\{\mathrm{U}_{\alpha} \mid \alpha \in \triangle\right.$ and $\left.\mathrm{U}_{\alpha} \in \tau\right\}$ be an open cover of $\mathrm{A}$. Then $\left\{\mathrm{U}_{\alpha} \mid \alpha \in \Delta\right\} \cup(\mathrm{X}-\mathrm{A})$ is a $\tau$-open cover of $\mathrm{X}$ and hence there exists a $\tau$-open precise $\tau$-locally finite refinement $\left\{\mathrm{V}_{\alpha} \mid \alpha \in \triangle\right\} \cup\{\mathrm{V}\}\left(\mathrm{V}_{\alpha} \subseteq \mathrm{U}_{\alpha}\right.$ and $\left.\mathrm{V} \subseteq \mathrm{X}-\mathrm{A}\right)$ such that $\mathrm{X}-\left[\mathrm{V} \cup\left(\mathrm{U}_{\alpha \in \Delta} \mathrm{V}_{\alpha}\right)\right] \in \mathcal{I}$. Now $\mathrm{A}-\cup_{\alpha \in \Delta} \cup \mathrm{V}_{\alpha}=$ $A-\left[V \cup\left(\cup_{\alpha \in \Delta} V_{\alpha}\right)\right] \subseteq X-\left[V \cup\left(\cup_{\alpha \in \Delta}^{\cup} V_{\alpha}\right)\right]$, hence $A-\bigcup_{\alpha \in \Delta} V_{\alpha} \in \mathcal{I}$ by the heredity of $\mathcal{I}$.

We see from Theorem IV.3 that the example of Zahid [1] of a closed subset of an H-closed space (and hence an $\mathcal{N}(\tau)$-paracompact Hausdorff space) which is not a para-H-closed subspace, is an example of an $\mathcal{N}(\tau)$-paracompact subset (and hence an $\mathcal{N}(\tau)$-paracompact subspace) which is not a para-H-closed subspace

THEOREM IV.4. Let $(\mathrm{X}, \tau, \mathcal{I})$ be a Hausdorff space. If $\mathrm{A} \subseteq \mathrm{X}$ is an $\mathcal{I}$-paracompact subset, then $\mathrm{A}$ is $\tau^{*}$-closed.

PROOF. Let $\mathrm{x} \in \mathrm{X}-\mathrm{A}$. For each $\mathrm{y} \in \mathrm{A}$, let $\mathrm{U}_{\mathrm{y}} \in \tau(\mathrm{x}), \mathrm{V}_{\mathrm{y}} \in \tau(\mathrm{y})$ such that $\mathrm{U}_{\mathrm{y}} \cap \mathrm{V}_{\mathrm{y}}=\emptyset$ and note that $\mathrm{x} \notin \mathrm{Cl}\left(\mathrm{V}_{\mathrm{y}}\right)$ Now $\left\{\mathrm{V}_{\mathrm{y}} \mid \mathrm{y} \in \mathrm{A}\right\}$ is a $\tau$-open cover of $\mathrm{A}$ and hence there exists a precise $\tau$-open $\tau$ locally finite refinement $\left\{V_{y}^{\prime} \mid y \in A\right\}$ of $\left\{V_{y} \mid y \in A\right\}$ such that $A-\cup_{y \in A} V_{y}^{\prime}=I \in \mathcal{I}$. Now $x \notin C l\left(V_{y}^{\prime}\right)$ for each y implies $\mathrm{x} \notin \underset{\mathrm{y} \in \mathrm{A}}{\cup} \mathrm{Cl}\left(\mathrm{V}_{\mathrm{y}}^{\prime}\right)=\mathrm{Cl}\left(\cup_{\mathrm{y} \in \mathrm{A}} \mathrm{V}_{\mathrm{y}}^{\prime}\right)$. Let $\mathrm{U}=\mathrm{X}-\mathrm{Cl}\left(\cup_{\mathrm{y} \in \mathrm{A}} \mathrm{V}_{\mathrm{y}}^{\prime}\right)$ and let $\mathrm{J}=\mathrm{A}-\mathrm{Cl}\left(\cup_{\mathrm{y} \in \mathrm{A}} \mathrm{V}_{\mathrm{y}}^{\prime}\right) \subseteq \mathrm{A}-$ $\left.\bigcup_{y \in A} V_{y}^{\prime}\right) \subseteq A-\bigcup_{y \in A} V_{y}^{\prime}=I$. Then $U-J \in \tau^{*}(x)$ and $(U-J) \cap A=\emptyset$, hence $A$ is $\tau^{*}$-closed.

The following example exhibits a $\{\emptyset\}$-paracompact subspace (and hence para-H-closed subspace) which is not an $\mathcal{N}(\tau)$-paracompact subset, thus showing that none of the arrows in Figure 1 are reversible and that "N( $\mathcal{N}$-paracompact subset" and "para-H-closed subspace" are independent concepts.

EXAMPLE. Let $X$ denote the real numbers and let $Q$ denote the rational numbers. Let $\tau$ be the topology generated by taking the usual open subsets and $\{\{q\} \mid q \in Q\}$ as a subbase. Now $Q$ is discrete and hence paracompact as a subspace, but $Q$ is not $\tau^{*}(\mathcal{N}(\tau))\left(=\tau^{\alpha}\right)$ closed and hence not an $\mathcal{N}(\tau)$ paracompact subset.

Let $(\mathrm{X}, \tau)$ be a topological space. It is well known that for every $\mathrm{A} \subseteq \mathrm{X}, \mathrm{A}^{*}(\mathcal{M}(\tau))$ is regular closed [5]. More generally, it follows from Theorems 3.2 and 3.3 of [9] that if $\mathcal{I}$ is a compatible ideal on $\mathrm{X}$ with $\mathcal{N}(\tau) \subseteq \mathcal{I}$, then $\mathrm{A}^{*}(\mathcal{I})$ is regular closed. This fact is used in the following decomposition theorem for $\mathcal{I}$ paracompact spaces.

THEOREM IV.5. Let $(\mathrm{X}, \tau, \mathcal{I})$ be an $\mathcal{I}$-paracompact space with $\mathcal{I} \sim \tau$ and $\mathcal{N}(\tau) \subseteq \mathcal{I}$ Then $\mathrm{X}=$ $\mathrm{A} \cup \mathrm{I}$ where $\mathrm{A}$ is a regular closed almost paracompact subspace (i.e. $(\mathrm{A}, \tau \mid \mathrm{A})$ is $\mathcal{N}(\tau \mid \mathrm{A})$-paracompact) and $I \in \mathcal{I}$. If $(X, \tau)$ is Hausdorff, then $A$ is para-H-closed.

PROOF. Since $\mathcal{I} \sim \tau, \mathrm{X}-\mathrm{X}^{*} \in \mathcal{I}$ and from the above remarks we have that $\mathrm{X}^{*}$ is regular closed. We let $\mathrm{A}=\mathrm{X}^{*}$ and $\mathrm{I}=\mathrm{X}-\mathrm{X}^{*}$. Note that $\mathrm{X}-\mathrm{X}^{*}=U\{\mathrm{U} \in \tau \mid \mathrm{U} \in \mathcal{I}\}$, and since $\mathrm{X}^{*}=\mathrm{Cl}\left(\operatorname{Int}\left(\mathrm{X}^{*}\right)\right)$ we have that $\mathcal{I} \mid \mathrm{X}^{*}$ is $\tau \mid \mathrm{X}^{*}$-boundary. Now by Theorem IV.3, $\mathrm{X}^{*}$ is an $\mathcal{I}$-paracompact subspace, i.e. $\mathrm{X}^{*}$ is an $\mathcal{I} \mid \mathrm{X}^{*}$-paracompact subspace. Also observe that since $\mathrm{X}^{*}$ is regular closed, we have $\mathcal{N}(\tau) \mid \mathrm{X}^{*}=\mathcal{N}\left(\tau \mid \mathrm{X}^{*}\right)$ Thus we have $\mathcal{I} \mid \mathrm{X}^{*}$ is a $\tau \mid \mathrm{X}^{*}$-boundary ideal on $\mathrm{X}^{*}$ with $\mathcal{N}\left(\tau \mid \mathrm{X}^{*}\right) \subseteq \mathcal{I} \mid \mathrm{X}^{*}$ and hence, by Corollary II.12, $\left(X^{*}, \tau \mid X^{*}\right)$ is almost paracompact as a subspace. If $(X, \tau)$ is Hausdorff, then $X^{*}$ is Hausdorff and hence is para-H-closed.

COROLLARY IV.6. Let $(\mathrm{X}, \tau)$ be an $\mathcal{M}(\tau)$-paracompact space. Then $\mathrm{X}=\mathrm{A} \cup \mathrm{I}$ where $\mathrm{A}$ is a regular closed almost paracompact subspace and $\mathrm{I}$ is meager. If $(\mathrm{X}, \tau)$ is Hausdorff then $\mathrm{A}$ is para-Hclosed as a subspace.

PROOF. It is well known [6, Banach Category Theorem] that $\mathcal{M}(\tau) \sim \tau$. The result then follows immediately from Theorem IV 5

ACKNOWLEDGMENT. The second and third authors received partial support through an East Central University Research Grant 


\section{REFERENCES}

[1] ZAHID, M I., Para-H-closed spaces, locally para-H-closed spaces and their minimal topologies, Ph.D dissertation, Univ. of Pittsburgh, 1981

[2] SINGAL, M. K. and ARYA, S. P., On m-paracompact spaces, Math. Ann., 181 (1969), 119-133.

[3] JANKOVIĆ, D. and HAMLETT, T.R., New topologies from old via ideals, Amer. Math. Monthly, Vol. 97, No. 4 (April, 1990), 295-310.

[4] NJÅSTAD, O., Remarks on topologies defined by local properties, Avh. Norske Vid.-Akad. Oslo I (N.S.), 8 (1966), 1-16.

[5] VAIDYANATHASWAMY, R., The localization theory in set-topology, Proc. Indian Acad. Sci., 20 (1945), 51-61.

[6] OXTOBY, J.C., Measure and Category, Springer-Verlag, 1980.

[7] HAMLETT, T.R. and JANKOVIĆ, D., Ideals in topological spaces and the set operator $\psi$, Boll. $U$. M. I., (7) 4-B (1990), 863-874.

[8] NEWCOMB, R.L., Topologies which are compact modulo an ideal, Ph.D. dissertation, Univ of Cal. at Santa Barbara, 1967.

[9] JANKOVIĆ, D. and HAMLETT, T.R., Compatible extensions of ideals, Boll. U.M.I. (7), 6-B (1992), 453-465.

[10] CROSSLEY, S.G. and HILDEBRAND, S.K., Semi-topological properties, Fund. Math., LXXIV (1972), 233-254.

[11] HAMLETT, T.R. and ROSE, D , *-topological properties, Internat. J. Math. \& Math. Sci., Vol. 13, No. 3 (1990), 507-512.

[12] JANKOVIĆ, D., On topological properties defined by semi-regularization topologies, Boll. U.M.I., 2-A, (1983).

[13] HAMLETT, T.R. and JANKOVIĆ, D., Compactness with respect to an ideal, Boll. U.M.I. (7), 4-B (1990), 849-862.

[14] MICHAEL, E., Another note on paracompact spaces, Proc. Amer. Math. Soc., Vol. 3 (1957), 822828.

[15] SINGAL, M.K. and SINGAL, ASHA RANI, Almost continuous mappings, Yokohama Math. J., 16 (1968), 63-73.

[16] WILLARD, STEPHEN, General Topology, Addison Wesley, Reading, 1970

[17] DIEUdONNÉ, J., Une généralization des espaces compacts, J. Math. Pures Appl., Vol. 23 (1944), 67-76.

[18] BERRI, M.P., PORTER, J.R., and STEPHENSON, JR., R.M., A survey of minimal topological spaces, Proc. Kanpur Topological Conf. 1968, General Topology and its Relations to Modern Analysis and Algebra, Vol. III, Academic Press, New York, 1970.

[19] AULL, C.E., Paracompact subsets, Proc. of the Second Prague Topological Symposium (1966), 4551.

[20] HAMLETT, T.R., JANKOVIĆ, DRAGAN, and ROSE, DAVID, Countable compactness with respect to an ideal, Math. Chron. 20 (1991), 109-126. 


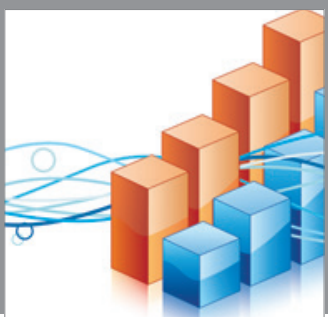

Advances in

Operations Research

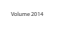

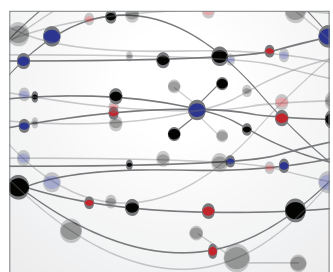

\section{The Scientific} World Journal
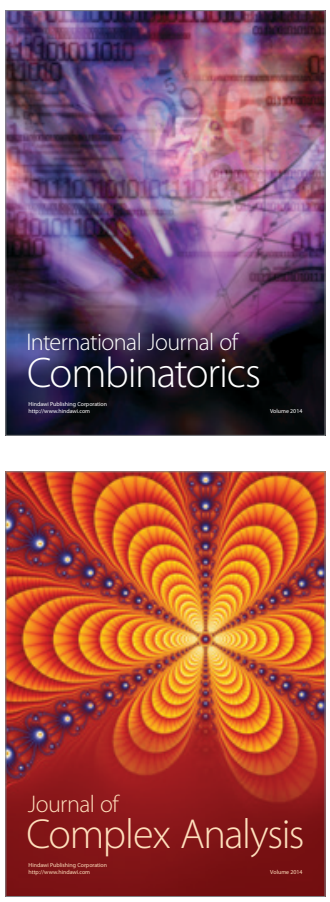

International Journal of

Mathematics and

Mathematical

Sciences
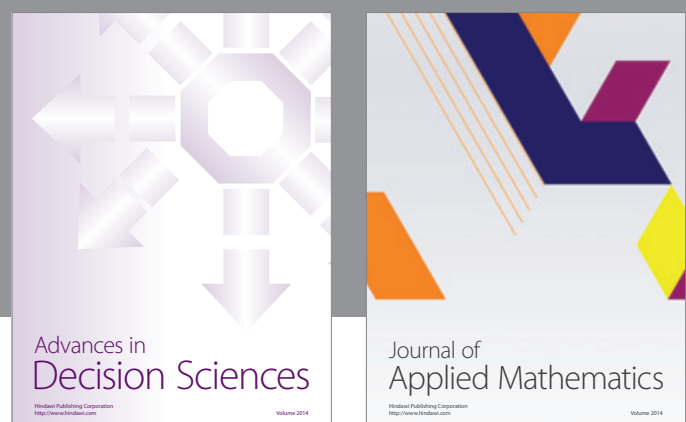

Journal of

Applied Mathematics
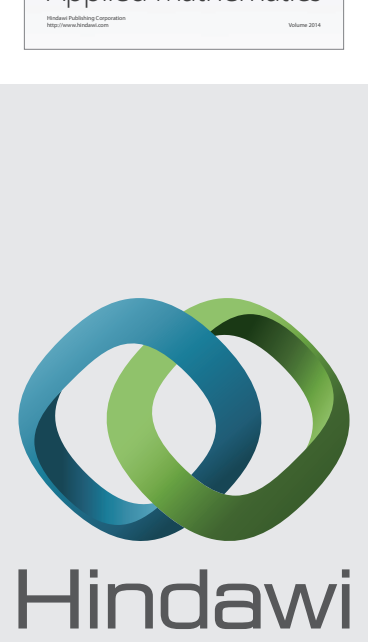

Submit your manuscripts at http://www.hindawi.com
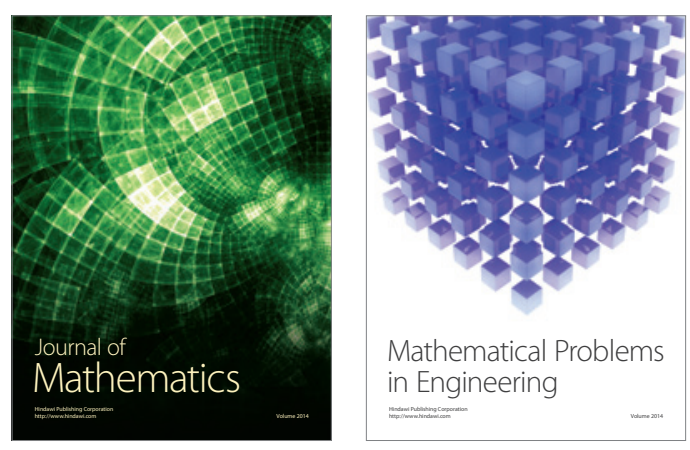

Mathematical Problems in Engineering
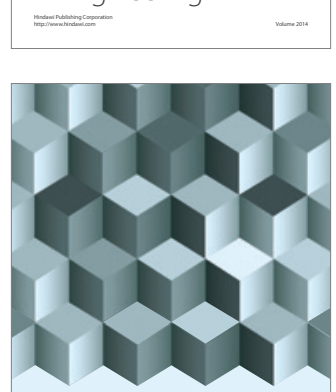

Journal of

Function Spaces
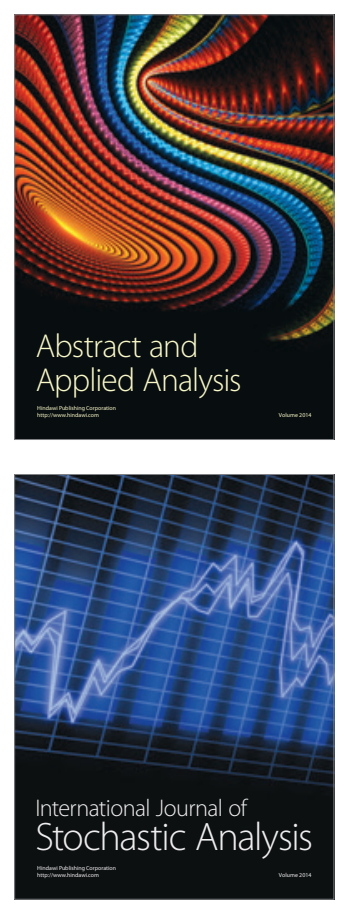

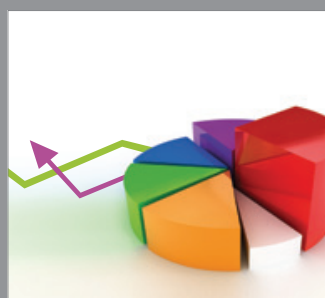

ournal of

Probability and Statistics

Promensencen
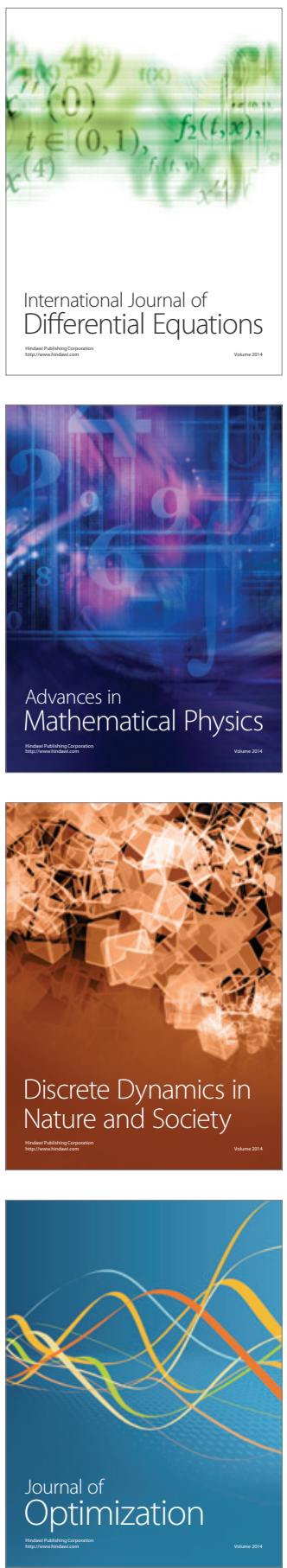